\title{
Whole-exome sequencing and microRNA profiling reveal PI3K/AKT pathway's involvement in juvenile myelomonocytic leukemia
}

\author{
Saad M Khan ${ }^{1}$, Jason E Denney ${ }^{2}$, Michael $X$ Wang $^{2,3, *}$ and Dong $\mathrm{Xu}^{1,3, *}$ \\ 1 Informatics Institute and C. S. Bond Life Sciences Center, University of Missouri, Columbia, MO 65211, USA \\ 2 Department of Pathology and Anatomical Sciences, University of Missouri, Columbia, MO 65211, USA \\ ${ }^{3}$ Department of Computer Science, University of Missouri, Columbia, MO 65211, USA \\ * Correspondence: wangmx@missouri.edu, xudong@missouri.edu
}

Received May 26, 2017; Revised July 27, 2017; Accepted July 27, 2017

\begin{abstract}
Background: Clinical studies and genetic analyses have revealed that juvenile myelomonocytic leukemia (JMML) is caused by somatic and/or germline mutations of genes involved in the RAS/MAPK signalling pathway. Given the vastly different clinical prognosis among individual patients that have had this disease, mutations in genes of other pathways may be involved.

Methods: In this study, we conducted whole-exome and cancer-panel sequencing analyses on a bone marrow sample from a 2-year old juvenile myelomonocytic leukemia patient. We also measured the microRNA profile of the same patient's bone marrow sample and the results were compared with the normal mature monocytic cells from the pooled peripheral blood.

Results: We identified additional novel mutations in the PI3K/AKT pathway and verified with a cancer panel targeted sequencing. We have confirmed the previously tested PTPN11 gene mutation (exon 3 181G $>$ T) in the same sample and identified new nonsynonymous mutations in NTRK1, HMGA2, MLH3, MYH9 and AKT1 genes. Many of the microRNAs found to be differentially expressed are known to act as oncogenic MicroRNAs (onco-MicroRNAs or oncomiRs), whose target genes are enriched in the PI3K/AKT signalling pathway.

Conclusions: Our study suggests an alternative mechanism for JMML pathogenesis in addition to RAS/MAPK pathway. This discovery may provide new genetic markers for diagnosis and new therapeutic targets for JMML patients in the future.
\end{abstract}

Keywords: single-cell; RNA-Seq; differential expression

\begin{abstract}
Author summary: We conducted whole-exome sequencing and microRNA profiling to analyze a patient of juvenile myelomonocytic leukemia. Our sequencing data and computational studies identified novel mutations in NTRK1, HMGA2, $M L H 3, M Y H 9$, and $A K T 1$ genes, which can potentially be used as diagnostic biomarkers and therapeutic targets. We suggest an alternative mechanism for JMML pathogenesis in addition to the RAS/MAPK pathway. We also identified a number of differentially expressed microRNAs that are known to act as oncogenic MicroRNAs in the patient sample. Our work provides an example of precision medicine to characterize the rare disease using a single-patient data.
\end{abstract}

\section{INTRODUCTION}

Juvenile myelomonocytic leukemia (JMML) is a rare but aggressive hematopoietic malignancy of early childhood. It is characterized by the overproduction of monocytic and granulocytic cells that circulate in the blood and infiltrate internal organs including the spleen, liver and 
other tissues. It is estimated to occur in 1.2 cases per million persons worldwide. The median age of children diagnosed with JMML is 2 years. Clinically, patients usually present with fever, leucocytosis, thrombocytopenia, and splenomegaly. The World Health Organization (WHO) has categorized JMML as an overlap myelodysplastic syndrome/myeloproliferative neoplasm (MDS/ MPN) and the diagnostic criteria have been defined based on clinical features, hematologic parameters, and genetic lesions. The prognosis of JMML is usually poor, and the only known effective treatment option is hematopoietic stem cell transplantation (HSCT). Treatment failure occurs in half of the patients due to disease relapse or transformation to acute myeloid leukemia (AML) [1].

Significant progress in understanding the molecular pathogenesis of JMML has been achieved by identifying the association of specific clinical syndromes with the genetic alterations in the RAS/MAPK signalling pathway, and by establishing the animal models with specific genetic lesions that mimic the disease [2]. The majority of such mutations have been found to occur in genes encoding proteins of the RAS/MAPK signalling pathway [3]. Mutations in NRAS, KRAS [4] and NF1 [5] in the RAS/MAPK signalling pathway have been known to be initiating events for JMML. Germline mutations in PTPN11 have also been reported in earlier studies in around $50 \%$ of the patients [6]. Some of the patients with nonsyndromic JMML have been reported to have somatic mutations in those genes. Homozygous CBL mutations have also been previously reported in around $15 \%$ of the cases [3]. Together, nearly $90 \%$ of the JMML patients have been found to carry somatic or germline mutations involving RAS/MAPK signalling pathway. Other mutations that are assumed to be secondary mutations and have only been reported in a few cases of JMML include ASXL1 [7], FLT3 [8], SRSF2 [9], SETBP1 and JAK3 [10]. Like every other malignancy, JMML also shows aberrant methylation patterns, with methylation being particularly enriched in $\mathrm{CpG}$ islands [11]. The microRNA profiles of JMML remain unexplored. Although it is now clear that JMML is fundamentally a disease of hyperactive RAS/ MAPK signalling, the targeted therapy towards the signalling relay proteins in this pathway has been largely unsuccessful [12].

In this study we utilized next-generation whole-exome sequencing strategy, which is an important technique with high coding region coverage and a high rate of phenotype association. This approach has been successfully used to identify important genomic variations in a number of diseases including driver mutations in cancer and germline mutations in inherited diseases $[10,13]$. Here, we report several novel mutations that are involved in the $\mathrm{P} 13 \mathrm{~K} / \mathrm{AKT}$ signalling pathway, which have not been reported previously in JMML patients. We hypothesize that the mutation in the P13K/AKT pathway along with differential regulation of microRNAs could be an alternative mechanism for JMML pathogenesis. Interestingly, several kinases in this pathway can be targeted with existing drugs that provide an alternative therapeutic potential for this deadly disease.

\section{RESULTS}

A bone marrow sample from a 2-year old juvenile myelomonocytic leukemia patient was used in this study for whole-exome, cancer-panel, and small RNA sequencing analysis. Exome analysis revealed some mutations that were damaging in nature and were confirmed by cancer panel, while smRNA-seq indicates roles of oncomiRs in JMML.

\section{Exome and cancer-panel analysis}

The whole-exome sequencing generated from the JMML patient bone marrow sample had around 39 million reads, of which $99 \%$ were mapped to the human genome using default settings in BWA [14]. Of those, $98 \%$ were proper pairs and around $90 \%$ were uniquely mapped as shown in Table 1 . The mapping criterion used to identify whether a read was uniquely mapped or not is the mapping quality (QS > 30). We were able to identify 126,835 variant calls in the JMML patient bone marrow leukemia cell DNA using the Mercury pipeline [15]. Of these, only 6,411 variants were able to pass the variant filtration step in Atlas tools (as a part of the Mercury pipeline). In order to identify the novel mutations from the variants, we first removed the SNPs recorded in dbSNP and 1,000 genomes project databases. Then we excluded synonymous SNPs and SNPs from intronic and non-coding regions. We were able to identify nonsynonymous mutations in NTKR1, ITPR 3, PTPN11, MLH3, AKT1, HMGA2, MYH9, and AR genes with average $30 \times$ coverage. The mutations we identified in whole-exome sequencing were further verified by a cancer panel targeted sequencing with $100 \times$ coverage of 573 genes (derived from the Sanger

Table 1 Alignment metrics for whole-exome and targeted cancer-panel sequencing

\begin{tabular}{llllll}
\hline Sequencing type & $\begin{array}{l}\text { Total no of reads } \\
\left(10^{6}\right)\end{array}$ & $\begin{array}{l}\text { Mapped Reads } \\
\left(10^{6}\right)\end{array}$ & $\begin{array}{l}\text { Proper pairs } \\
\left(10^{6}\right)\end{array}$ & $\begin{array}{l}\text { PCR duplicates } \\
\left(10^{6}\right)\end{array}$ & $\begin{array}{l}\text { Uniquely mapped } \\
\left(10^{6}\right)\end{array}$ \\
\hline Whole exome & 39.41 & $38.94(98.82 \%)$ & $38.74(98.30 \%)$ & $1.51(3.84 \%)$ & $35.58(90.30 \%)$ \\
Cancer panel & 14.20 & $13.90(97.91 \%)$ & $13.81(97.28 \%)$ & $1.23(8.66 \%)$ & $12.92(91.03 \%)$ \\
\hline
\end{tabular}


cancer gene list). The Mercury pipeline was again used to call variants. A total of 25,633 variants were called, only 6,335 of which passed the variant filtration in the Mercury pipeline. The following mutations were confirmed by both exome and cancer-panel sequencing, namely ITPR3 (chr6:33651070, G > A, Exon35, A1562 > T), PTPN11 $($ chr12:112888165, G $>\mathrm{T}$, Exon3, D61 > Y $), A K T 1$ (chr14:105239869, G > A, Exon9, R251 > C), MLH3 (chr14:75514537, A > T, Exon2, F608 > I), and MYH9 (chr22:36685257, C $>$ A, Exon32, K1477 $>$ N). Other mutations including $A R$ (chrX: 66765152, $\mathrm{T}>\mathrm{A}$, Exon1, L55 > Q), NTRK1 (chr1:156843653, C > T, Exon8, T360 $>\mathrm{M}$ ) and mutations in $H M G A 2$ gene (chr12:66260663, 66260665, C > G, G > T, Exon2, Q7 > H, Q113>K) were only seen in the whole-exome sequencing as these genes are not part of the cancer panel.

PTPN11 gene mutation had already been found in the JMML patient tested in this research in a diagnosis test, and we were able to see the same mutation in both exome and cancer-panel sequencing. There is no discordant event between the mutations identified in the same genes in both sequencing methods. Table 2 shows the genes, which had nonsynonymous mutations along with their locations in the genomic regions and gene body, and their resulting amino acid change. SIFT and Provean [16] predictions suggest that some mutations have damaging effects and are deleterious in nature for the functions of the coded proteins as shown in Table 2. Notably, the mutation of $A K T 1$ results in significant structural disruption and the mutation is shown encircled in Figure 1 [17], while other mutations shown in this figure did not pass the variant filtration step. AKT1 (chr14:105239869, $\mathrm{G}>\mathrm{A}$, Exon9, R251 > C) mutation results from SusPect [18] and HOPE [19] indicated a structural disruption due to this point mutations. Results from HOPE indicate that the mutation lies in an Interpro [20] domain, i.e., the protein kinase domain (IPR000719). The domain is enriched with GO terms like ATP binding (GO: 0005524) and protein kinase activity (GO: 0004672). A homology model of the AKT1 mutation based on the template [PDB: 3096] with 97\% identity is shown in Figure 2A superimposed with the native structure.

Ligplot results of the native structure of AKT1 (3O96) around ARG251 are shown and compared with the mutated residue CYS189 of AKT1 in Figure 2B and 2C. Ligplot results indicate a change in hydrogen bonding and hydrophobic contacts of CYS189 when compared with ARG251. Some of these contacts tend to lie in the active site regions of protein as predicted by Motif Scan results, e.g., PHE407, which shares a hydrogen bond with neighbours of ARG251 in the native structure but did not do so in the JMML mutated AKT1 protein. Also there was an overall change in the net charges due to the mutation of ARG251 to CYS189, which may have also affected active sites of the mutated protein. The importance of the $A K T 1$ mutation as well as other mutations like in PTPN11, MYH9 and MLH3 is also shown in Supplementary Figure S1, where the position is highly conserved in other species. This result strongly suggests the ARG is an important amino acid residue for AKT1 enzyme activity.

\section{Small RNA analysis}

From our small RNA-seq analysis we identified differentially expressed microRNAs in comparison to JMML leukemia cells with monocytes from normal adult pooled peripheral blood samples (Supplementary Tables S1-S3). A total of 230 microRNAs showed significant $p$-value $(\leqslant 0.01)$ and fdr $(\leqslant 0.01)$, of which 76 microRNAs showed $>2$-fold upregulation (Supplementary Table S1) and 66 microRNAs showed $>2$-fold downregulation (Supplementary Table S2) with respect to adult monocytes. Many of the microRNAs that were found to be differentially expressed were associated with some types of cancer, and some of them are categorized under the class of oncomiRs. These oncomiRs have been known to play a vital role in tumorigenesis, malignant transformation, and metastasis [22]. We found hsa-miR-19a-3p ( $\log 2$-fold-change $=2.33)$ and hsa-miR-19b-3p $(\log 2-$ fold-change $=4.34$ ) were upregulated in JMML compared with normal monocytes, and they were also upregulated in several other types of cancers such as gastric cancer, lung cancer, osteosarcoma and squamous cell carcinoma $[23,24]$. Likewise, hsa-miR-15a-5p (log2fold-change $=2.26)$ and hsa-miR-15b-3p $(\log 2$-foldchange $=3.78)$ another well classified oncomiR family were found specifically upregulated in JMML. While the miR-15 family has been known to be down regulated in chronic lymphocytic leukemia $[25,26]$, we found it to be significantly upregulated in JMML. Unlike many other cancers in which hsa-miR-145 [27,28] and hsa-miR-126 $[29,30]$ are mostly down-regulated, JMML showed an upregulation for these two miRNAs. Many of the downregulated miRNAs in JMML show similar properties in other types of cancers, e.g., hsa-miR-193a [31] and hsamiR-125a [32], which have been found to be downregulated in most cancers. Lastly, microRNAs like hsalet $7 \mathrm{~b} / \mathrm{c} / \mathrm{d} / \mathrm{e} / \mathrm{f}$ were also downregulated in JMML, although the Let-7 family of microRNAs is related to development, but in cancer their major function is related to tumoursuppression. Let-7 family is known to regulate RAS expression in humans and is also known to target the HMGA2 gene. The observed downregulation of Let-7 could be associated with over-expression of RAS genes, which has been observed in some cancers [33]. Many of these microRNAs show enrichment in important cell signalling pathways that show extensive "cross-talking" 


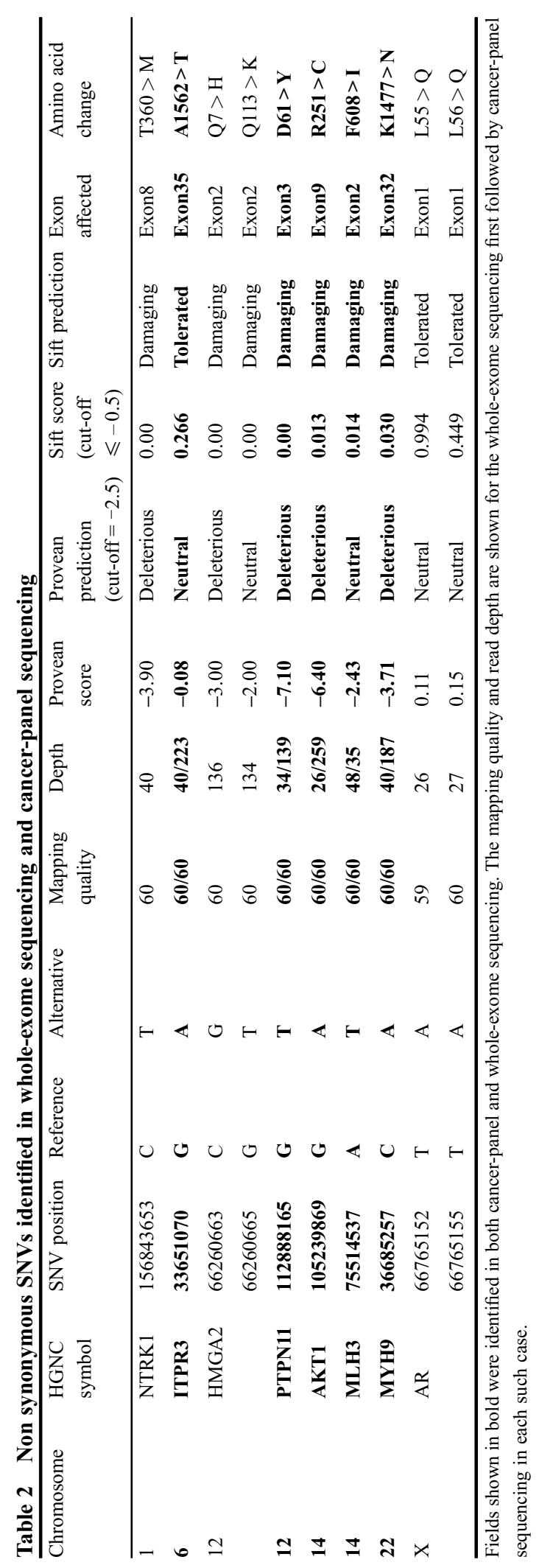




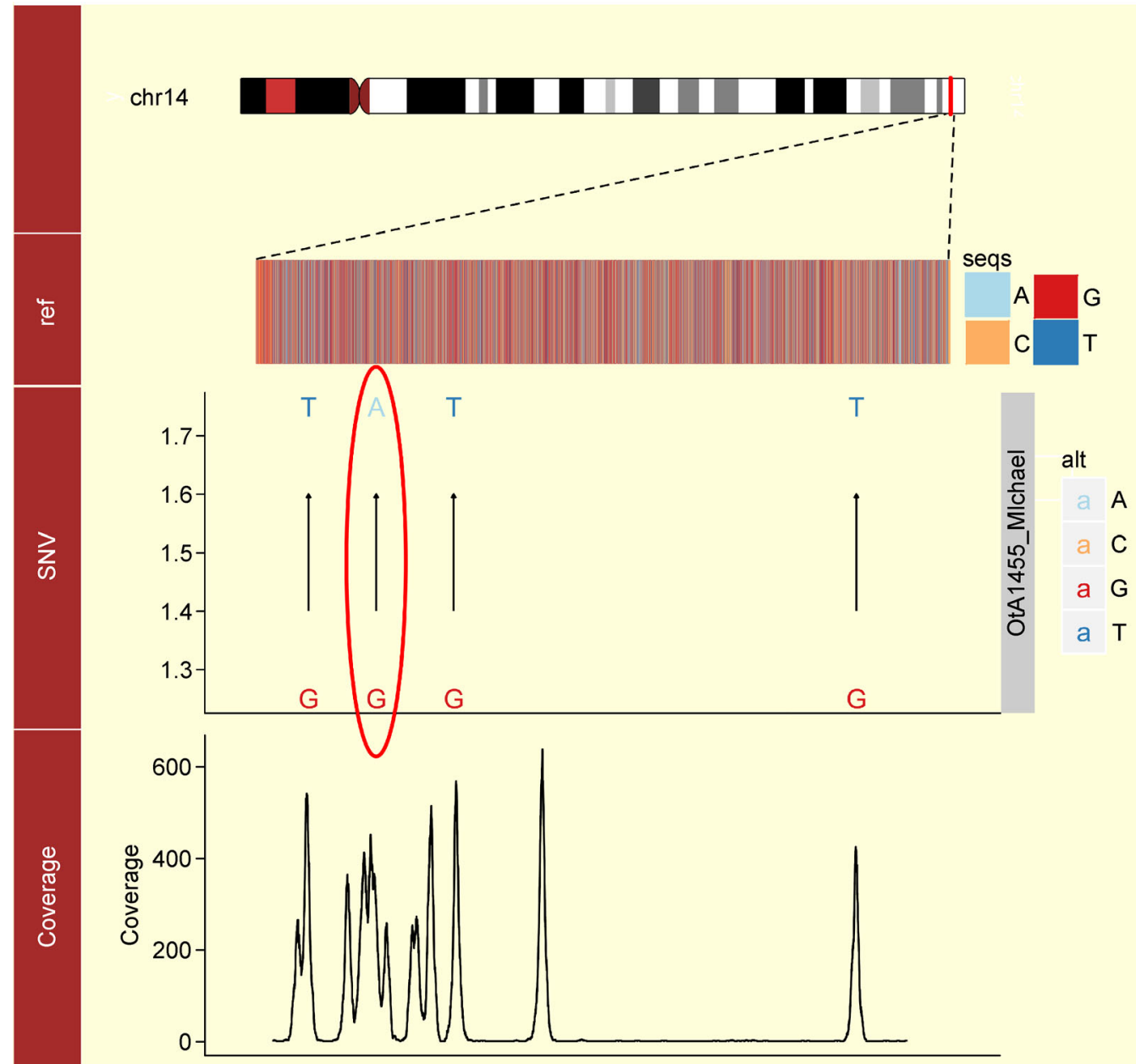

Figure 1. Alignment for the AKT1 gene is shown using the cancer panel vef files and alignment files. The first panel shows the location of the mutation on the hg19 assembly chromosome 14. The second panel shows the bar indicating reference sequence. The third panel indicates the nonsynonymous mutation encircled in red. The last panel shows the coverage of cancer-panel sequencing in the AKT1 gene including the nonsynonymous mutation. The mutation is present at exon9 in most transcripts of the $A K T 1$ gene. The figure was generated using ggbio [17] in $\mathrm{R}$.

with each other, e.g., mTOR pathway, RAS pathway and PI3K-AKT pathway. The enrichment profile of the most significant miRNA's based on $p$-value, fold change, and number of predicted gene targets is shown in Figure 3. The enrichment heat map for all upregulated and all downregulated microRNAs is shown in Supplementary Figures S2 and S3, respectively, both of which show enrichment in some cancer related pathways.

\section{DISCUSSION}

Most disease-causing mutations occur in the coding regions of the genome. Whole-exome sequencing helps identify such variants that are correlated with phenotypes with high accuracy. In this study we were able to identify novel mutations in JMML bone marrow leukemia cells. In addition, we also performed targeted cancer panel deep sequencing, which confirmed our observations from whole-exome sequencing. We were able to confirm widely reported and previously tested mutations in the PTPN11 gene (chr12:112888165, G> T, Exon3, D61 $>$ Y) in this study's JMML patient. PTPN11 played an important role in regulating the RAS/MAPK signalling pathway [6]. Another mutation of considerable importance is the AKT1 (chr14:105239869, G > A, Exon9, $\mathrm{R} 251>\mathrm{C})$ mutation. AKT1 gene codes for a serine/ threonine specific kinase, which plays a key role in multiple cellular processes, such as glucose metabolism, apoptosis, cell proliferation and cell migration [36]. The roles of $A K T 1$ in many human cancers (e.g., gastric, ovarian, squamous and lung) have also been widely reported [37]. To our knowledge, AKT1 mutation has not 


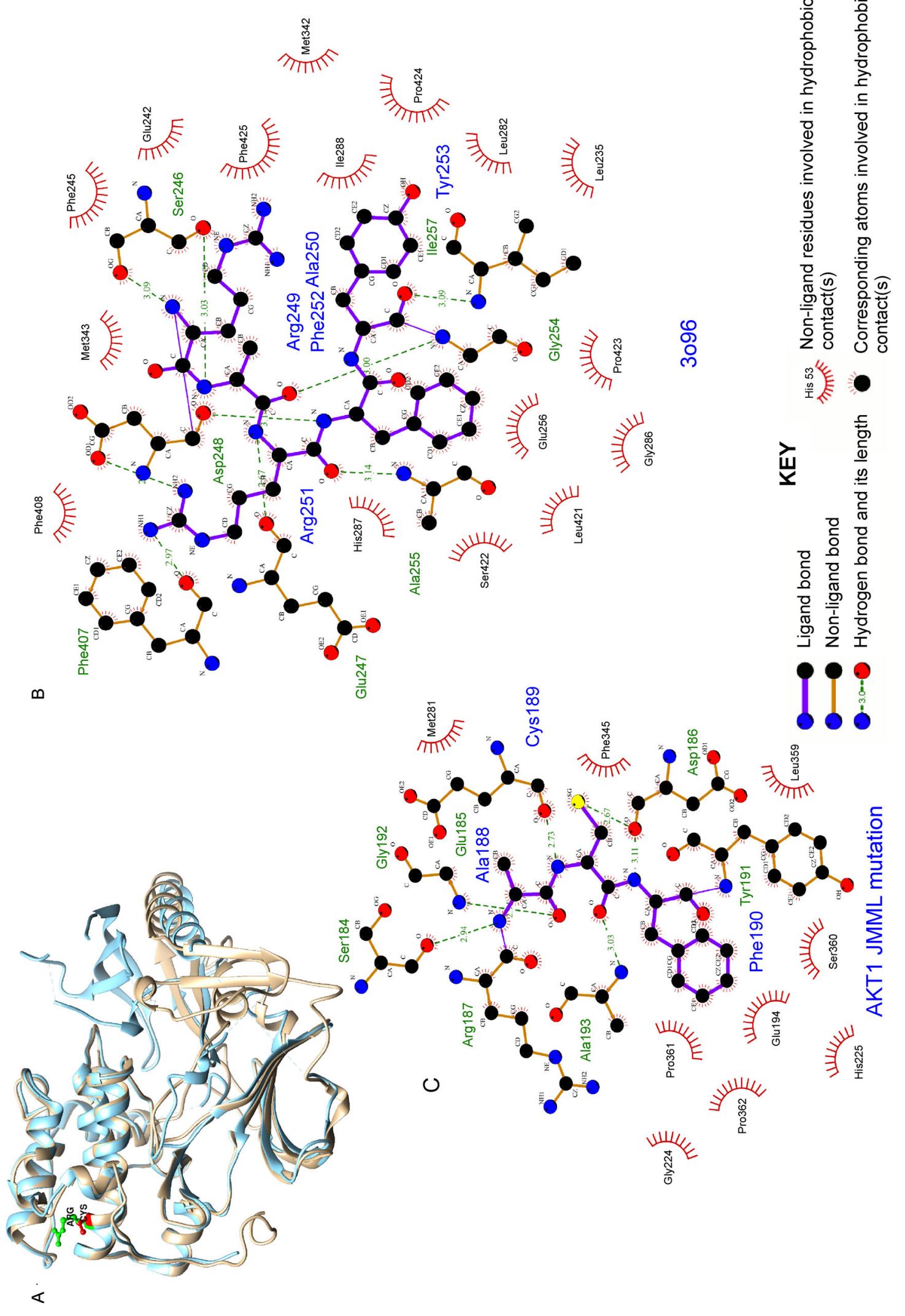

蒿望 $\frac{n}{0}$

व

势安

:

은 윽 을 宫

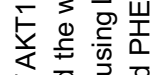

흘 웡

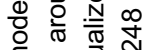

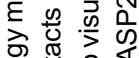

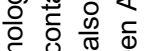

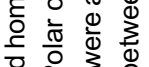

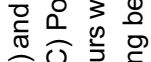

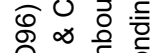

잉 홍

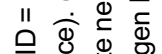

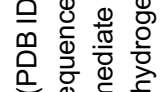

ब

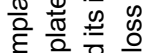

ब

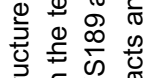

蛋 $\cong$ 更

등 \&

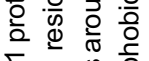

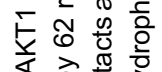

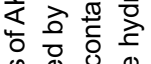

\& 0

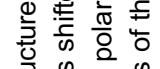

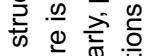

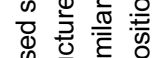

을 $\stackrel{5}{=}$.

亭 岸

क्षे 응 +

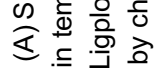

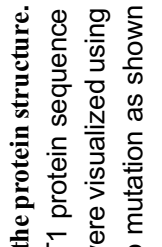

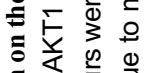

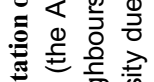

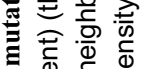

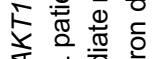

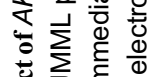

疋

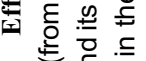

ن

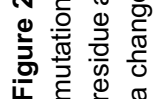






Figure 3. Pathway enrichment of microRNAs in JMML. Results from Diana-mirPATH v-3 [34] denoting enrichment of microRNAs in different pathways wherein microRNAs are clustered with respect to their log ( $p$-value) for enrichment. Only microRNAs with most significant $p$-values were taken. The pathway union method was used in Diana-miRPath v-3 to generate the heat map. The heat map of selected microRNAs was visualized using pheatmap [35]. Most microRNAs with significant $p$-values were upregulated; the significance of their fold-change is also shown in the heatmap.

been reported in JMML. MLH3 (chr14:75514537, A > T, Exon2, F608 > I) mutation was also identified in both whole-exome and cancer-panel sequencing. This gene belongs to the family of DNA mismatch repair genes and is involved in maintaining genomic integrity during replication. MLH3 mutation may be related to a high somatic mutation load in this particular patient [38]. Somatic mutations in these genes have also been widely reported but their evidence for a role in cancers remains unclear [39-41]. MYH9 (chr22:36685257, C > A, Exon32, K1477 $>$ N) gene plays an important role in cytokinesis, cell shape and specialized functions such as secretion and capping. Genetic mutations in $M Y H 9$ have been reported in lung cancer $[42,43]$. Other mutations that have a damaging effect and were only seen in whole- exome sequencing are in $H M G A 2$ gene $(\mathrm{Q} 7>\mathrm{H}$, $\mathrm{Q} 113>\mathrm{K})$ and NTRK1 gene $(\mathrm{T} 360>\mathrm{M})$. HMGA2, is an architectural protein and an essential component of enhanceosome [44] while NTRK1 is a neurotrophic tyrosine kinase receptor and phosphorylates members of the MAPK pathway. Mutations in both $H M G A 2$ and NTRK1 have also been implicated in cancers [44-46]. By comparing the microRNA expression profile of this JMML sample with the mature monocyte preparation, we were able to identify a group of microRNAs that are involved in different types of cancers. Of considerable importance is the microRNA-19, which is an oncomiR and is also known to target PTEN [47] in the PI3K/AKT signalling pathway. It has been observed that activation of $\mathrm{PI} 3 \mathrm{~K} / \mathrm{AKT}$ signalling plays an important role in stem cell 
cancers while the JMML is considered to be of hematopoietic stem cell origin [48]. Lastly, JMML microRNA profiles bear some similarities with other types of cancers in terms of microRNAs that are up/down regulated but also have features specific to JMML.

Signalling networks usually display a PI3K/AKT pathway and a RAS/MAPK pathway as independent parallel pathways, but these two pathways share multiple cross-talk points between each other as shown in Figure 4 [49]. Roles of both RAS and PI3K pathway have been found in some cancers and drugs or combinations of drugs targeting both pathways have been suggested. Our findings reveal that mutations in the $A K T 1$ gene could cause dysregulation in the RAS pathway due to the apparent cross-talk between the two pathways. From the results above, we are able to hypothesize that a mutation in $A K T 1$ affects the PI3K/AKT/mTOR signalling pathway activity that may also cause dysregulation in the RAS/MAPK pathway [50,51]. While the RAS pathway has been known to be the most common route for JMML cancer causation or proliferation, we emphasize here that
PI3K/AKT may serve as another alternative mechanism for JMML causation, which opens up the possibility of designing new inhibitors targeting both pathways as shown in Figure 4. Also, our identification of a number of microRNAs that are differentially expressed in JMML suggests a possible role of microRNAs as mediators or effectors in JMML initiation and progression. Some of these oncomiRs that were found to be upregulated in our analysis are known to interact with PTEN, whose mutations and/or silencing has been implicated in various cancers and is known to lead to hyperactive PI3K signalling.

$A K T 1$ plays an important role in cell growth, apoptosis, proliferation, transcription and cell migration. AKT1 exhibits these functions by mediating multiple signalling pathways including the $\mathrm{PI} 3 \mathrm{~K} / \mathrm{AKT} / \mathrm{mTOR}$ pathway. $A K T 1$ activation is associated with many malignancies including solid tumours and acute myeloid leukaemia. Current clinical trials demonstrate that the AKT1 inhibitors can be used to treat hematopoietic malignancies as well as solid tumours. The most promising inhibitors

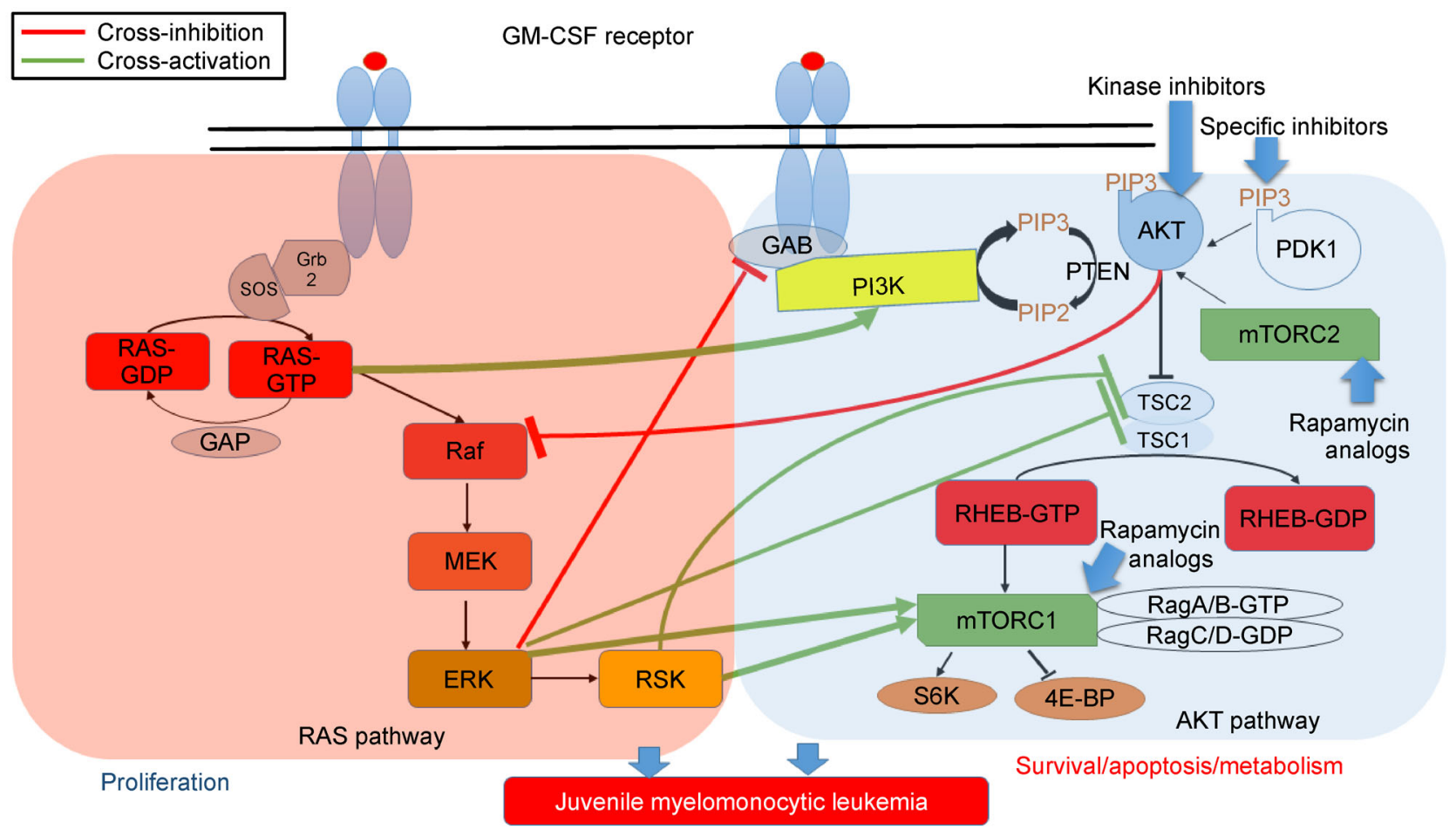

Figure 4. Possible Role of AKT in JMML Leukemogenesis. An alternative mechanism for JMML pathogenesis involving the role of newly identified heterozygous mutation in AKT1 as driving the JMML progression (modified from Mendoza et al. [49]). The RAS pathway and AKT pathway regulate each other with multiple modes of cross-talk most importantly cross-inhibition (red) and cross-activation (green). ERK phosphorylation of GAB and AKT phosphorylation of Raf are the key negative feedback loops of the two pathways. Ras, Raf, ERK, and RSK are some of the components that positively regulated the AKT pathway. TSC2 and mTORC are key integration points between two signalling pathways. Positive regulation of a protein is shown by an arrow while negative regulation is shown by blunt-ended line. Kinase inhibitors shown in bold blue arrows are known to target AKT protein. Rapamycin analogues are also known to target mTOR protein. The presence of AKT1 mutation in JMML provides interesting opportunity to use drugs to target this gene for JMML treatment. 
include perifosine (KRX-0401, Aeterna Zentaris/Keryx), MK-2206 (Merck), and GSK-2141795 (Glaxo-SmithKline). In addition, "pan-PI3K" inhibitors and dual PI3K/ mTOR inhibitors are in active clinical trials. In fact, two mTOR inhibitors, everolimus and temsirolimus, have been approved by Food and Drug Administration (FDA) to treat multiple types of solid tumours. From our studies, these drugs may potentially be used in treatment of JMML as an "off-label" mechanism [52,53]. Rare disorders like JMML provide a realistic future setting for personalized medicine using a single patient data. Our study highlights some of the ways in which multiple types of data can be used together to arrive at a possible treatment management plan. Use of personalized medicine or precision medicine in oncology based on patient specific genetics is now starting to be recognized [54]. Due to the burst of next-generation sequencing data, there has been a trend among clinicians to use genomic data to more precisely diagnose cancer, which can lead to a treatment of the cancer patient that is both effective and efficient. In rare diseases such as JMML, most of the personalized approaches rely on a very small subset of patients, and in most cases they tend to follow an N-of-1 trial approach in which the single patient is the entire trial [55]. Many times this N-of- 1 study is also executed in an $\mathrm{AB}$ quasi experimental way where the causality cannot be definitely demonstrated. When it comes to precision medicine of rare disorders, such experimental setups can be useful. Thus rare disorders like JMML in this report provide a model for personalized medicine using a single patient data. Our preliminary discovery may help identify new genetic markers for diagnosis and new therapeutic targets using specific kinase inhibitors or RNA interference (RNAi) for JMML treatment.

\section{CONCLUSIONS}

By using whole-exome sequencing, we have confirmed the existing mutation in the PTPN11 gene (chr12:112888165, G > T, Exon3) and discovered several novel mutations including AKT1 (chr14:105239869, $\mathrm{G}>\mathrm{A}$, Exon9) in our JMML patient. The microRNA profiling revealed the dysregulation in multiple microRNAs that were enriched in PI3K/AKT and other pathways. Extensive cross-talking between the newly identified PI3K/AKT pathway and the classical RAS/ MAPK pathway in JMML suggests a synergetic effect in JMML leukemogenesis. Our approach and sequencing data not only identified novel mutations that can potentially be used as a diagnostic biomarker and therapeutic target in JMML, but also provide a model to characterize the rare disease in the personalized precision medicine era.

\section{MATERIALS AND METHODS}

\section{Whole exome sequencing}

One human subject was involved in this research project. The DNA was extracted from patient blood and bone marrow samples. The authors (Dr. MICHAEL X. WANG and JASON DENNEY) have obtained an approval of Institutional review board of University of Missouri Health Science Center (IRB\# 1208122, HS IRB USE ONLY Approval Date: Aug 6, 2013) and signed a consent form from the parent. Genomic DNA was extracted from the bone marrow aspirate specimen of the 2-year old male patient with JMML after obtaining the IRB approval and a consent form signed by the parent. The libraries were prepared from fragmented DNA using the TruSeq DNA Library Preparation Kit (Illumina) and the enriched exome libraries were clonal amplified and sequenced using an Illumina HiSeq 2000 sequencer. The paired-end sequences were subject to quality control and trimmed using Fastqc [56]. The quality-controlled reads were then processed using a locally installed version of the Mercury pipeline [15]. The pipeline goes through several steps starting from mapping of the reads to a reference genome using BWA [14], which produces a BAM file. The reference assembly used in this process was GRCh37 obtained from the Human Genome Sequencing Center (https://www.hgsc.bcm.edu/). The aligned BAM file was further processed by tools like Picard (http://picard. sourceforge.net), SAMtools [57] and GATK [58] which further refined the BAM file by sorting, duplicatemarking, indel-realignment, base quality recalibration and indexing. The variants were called on the finished BAM file using Atlas-SNP and Atlas-Indel as part of the Atlas2 suite [59]. The called variants were finally annotated using Cassandra which combines the Annovar [60] output along with other public data sources like COSMIC (Catalogue of Somatic Mutations in Cancer) [61] to produce annotated vcf files.

\section{Cancer-panel sequencing}

In order to validate the mutations that we identified from whole-exome sequencing, we further conducted deep sequencing with $100 \times$ coverage of a cancer gene panel derived from the Sanger Institutes Cancer gene list [62]. Targeted sequencing of 533 cancer genes was performed using the Illumina HiSeq 2000 sequencer on the same DNA sample of this JMML patient. The reads obtained were subjected to quality control using Fastqc and analysed using the Mercury pipeline described in the exome analysis method. 


\section{Identifying nonsynonymous mutations}

In order to identify disease-associated mutations from both whole-exome sequencing and cancer-panel sequencing, we removed the variants that were common to Dbsnp (hg19 assembly), Dbsnp (grch37 assembly) and 1,000 genomes SNPs. This was achieved using the bedtools intersect program [63]. The vcf files for each of the above-mentioned data were obtained from the Human Genome Sequencing Center(https://www.hgsc.bcm.edu/). Once the list of mutations was obtained, it was run through the SIFT [16] algorithm to identify functionally important variants. Protein sequence of the mutated genes and conservation comparison was obtained using MutationTaster [64] and structural analysis was performed using HOPE [19] and SusPect [18]. Further homology modelling of AKT1 protein was carried out using 3096 PDB structure with SWISS-MODEL [65] and the protein structure was visualized using UCSF Chimera [66]. The polar contacts around the mutation and native residue were constructed using Ligplot+ [21].

\section{MicroRNA analysis}

To identify microRNAs that might play regulatory roles in gene expression of JMML, we extracted total RNA from the same patient's bone marrow aspirate sample. Illumina TruSeq small RNA preparation kits were used for generating small RNA libraries from total RNA. Strand specific paired-end RNA-seq data was obtained using TruSeq small RNA sequencing. The TruSeq adapters were removed using the bbduk program of the bbmap package (sourceforge.net/projects/bbmap/). The mate 2 of the paired-end RNA-seq fastq file was reversely complemented using the FASTX-Toolkit (http://hannonlab. cshl.edu/fastx_toolkit/). The reversely complemented mate2 was then combined with the mate1 fastq file. sRNAbench [67] a publicly available small RNA pipeline, was used for small RNA analysis. The mapping of all the reads was done against the GRCh37 human genome using sRNAbench after the genome had been indexed. After profiling expression of miRNA for JMML patient data we used publicly available peripheral blood monocyte data from 10 normal adults (GSE47189) of small RNA-seq to compare it with the JMML miRNA profile. The data was downloaded from NCBI GEO. Once the data was analysed using sRNAbench, counts were calculated using sRNAbenchDE for JMML sample and normal monocytes. Differential expression analysis was performed using both edgeR [68] and DESeq [69]. The microRNAs differentially expressed in both edgeR and DESeq were used for further analysis. Pathway enrichment for the microRNAs was done using miRPath [34] from the DIANA-tools webserver. A network of genes involved in cross-talk was constructed using Cytoscape 3.2 [70]. MicroRNA gene target information from miRTarBase Release 4.5 [71] was used along with the $p$-values and $\operatorname{logFC}$ values obtained from differential expression analysis for network visualization using Cytoscape for genes involved in cross-talk between RAS and PI3K/AKT pathway and their first degree neighbouring genes. AKT and RAS pathways were downloaded from KEGG as KGML/XML files and uploaded in Cytoscape. The genes shown in Figure 4 were selected along with first-degree neighbours and visualized. The network was merged with information of microRNA targets and microRNA expression. Log2-foldchange was used to do a continuous mapping of colour from green to red with green being upregulated and red being downregulated using vizmapper in Cytoscape. Two networks shown in Cytoscape are presented in Supplementary Figures S4 and S5.

\section{SUPPLEMENTARY MATERIALS}

The supplementary materials can be found online with this article at 10.1007/s40484-017-0125-2.

\section{ACKNOWLEDGEMENTS}

This work was partially supported by the Mizzou Advantage Program at the University of Missouri and National Institute of Health (R01-GM100701). The authors would like to thank the Informatics Core of the University of Missouri for providing the computing resources of this work. The authors would also like to thank Mr. Miqdad O. Dhariwala for providing access to and help with Canvas image manipulation software.

\section{COMPLIANCE WITH ETHICS GUIDELINES}

The authors Saad M Khan, Jason E Denney, Michael X Wang and Dong Xu declare they that have no conflict of interests.

All procedures performed in studies involving human participants were in accordance with the ethical standards of the institutional and/or national research committee and with the 1964 Helsinki declaration and its later amendments or comparable ethical standards.

\section{REFERENCES}

1. Arber, D. A., Orazi, A, Hasserjian, R., Thiele, J., Borowitz, M. J., Le Beau, M. M., Bloomfield, C. D., Cazzola, M., Vardiman, J. W. (2016) The 2016 revision to the WorldHealth Organization classification of myeloid neoplasms and acute leukemia. Blood, $127,2391-405$

2. Beurlet, S., Chomienne, C. and Padua, R. A. (2013) Engineering mouse models with myelodysplastic syndrome human candidate genes; how relevant are they? Haematologica, 98, 10-22

3. Loh, M. L. (2011) Recent advances in the pathogenesis and treatment of juvenile myelomonocytic leukaemia. Br. J. Haematol., 152, 677-687

4. Basu, T. N., Gutmann, D. H., Fletcher, J. A., Glover, T. W., Collins, 
F. S. and Downward, J. (1992) Aberrant regulation of ras proteins in malignant tumour cells from type 1 neurofibromatosis patients. Nature, 356, 713-715

5. Patil, S. and Chamberlain, R. S. (2012) Neoplasms associated with germline and somatic NF1 gene mutations. Oncologist, 17, 101116

6. Loh, M. L., Vattikuti, S., Schubbert, S., Reynolds, M. G., Carlson, E., Lieuw, K. H., Cheng, J. W., Lee, C. M., Stokoe, D., Bonifas, J. M., et al. (2004) Mutations in PTPN11 implicate the SHP-2 phosphatase in leukemogenesis. Blood, 103, 2325-2331

7. Sugimoto, Y., Muramatsu, H., Makishima, H., Prince, C., Jankowska, A. M., Yoshida, N., Xu, Y., Nishio, N., Hama, A., Yagasaki, H., et al. (2010) Spectrum of molecular defects in juvenile myelomonocytic leukaemia includes ASXL1 mutations. Br. J. Haematol., 150, 83-87

8. Gratias, E. J., Liu, Y. L., Meleth, S., Castleberry, R. P. and Emanuel, P. D. (2005) Activating FLT3 mutations are rare in children with juvenile myelomonocytic leukemia. Pediatr. Blood Cancer, 44, 142-146

9. Hirabayashi, S., Flotho, C., Moetter, J., Heuser, M.,Hasle, H., Gruhn, B., Klingebiel, T., Thol, F., Schlegelberger, B., Baumann, I., et al. (2012) Spliceosomal gene aberrations are rare, coexist with oncogenic mutations, and are unlikely to exert a driver effect in childhood MDS and JMML. Blood, 119, e96-e99

10. Sakaguchi, H., Okuno, Y., Muramatsu, H., Yoshida, K., Shiraishi, Y., Takahashi, M., Kon, A., Sanada, M., Chiba, K., Tanaka, H., et al. (2013) Exome sequencing identifies secondary mutations of $S E T B P 1$ and $J A K 3$ in juvenile myelomonocytic leukemia. Nat. Genet., 45, 937-941

11. Olk-Batz, C., Poetsch, A. R., Nöllke, P., Claus, R., Zucknick, M., Sandrock, I., Witte, T., Strahm, B., Hasle, H., Zecca, M., et al. (2011) Aberrant DNA methylation characterizes juvenile myelomonocytic leukemia with poor outcome. Blood, 117, 4871-4880

12. Lauchle, J. O. and Braun, B. S. (2010) Targeting RAS Signaling Pathways in Juvenile Myelomonocytic Leukemia (JMML). In Molecularly Targeted Therapy For Childhood Cancer. Houghton, P. J., Arceci, R. J., eds. pp. 123-138 New York: Springer

13. Yang, Y., Muzny, D. M., Reid, J. G., Bainbridge, M. N., Willis, A., Ward, P. A., Braxton, A., Beuten, J., Xia, F., Niu, Z., et al. (2013) Clinical whole-exome sequencing for the diagnosis of mendelian disorders. N. Engl. J. Med., 369, 1502-1511

14. Li, H. and Durbin, R. (2010) Fast and accurate long-read alignment with Burrows-Wheeler transform. Bioinformatics, 26, 589-595

15. Reid, J. G., Carroll, A., Veeraraghavan, N., Dahdouli, M., Sundquist, A., English, A., Bainbridge, M., White, S., Salerno, W., Buhay, C., et al. (2014) Launching genomics into the cloud: deployment of Mercury, a next generation sequence analysis pipeline. BMC Bioinformatics, 15, 30

16. Kumar, P., Henikoff, S. and Ng, P. C. (2009) Predicting the effects of coding non-synonymous variants on protein function using the SIFT algorithm. Nat. Protoc., 4, 1073-1081

17. Yin, T., Cook, D., Lawrence, M. (2012) ggbio: an R package for extending the grammar of graphics for genomic data. Genome Biol., 13, R77
18. Yates, C. M., Filippis, I., Kelley, L. A. and Sternberg, M. J. (2014) SuSPect: enhanced prediction of single amino acid variant (SAV) phenotype using network features. J. Mol. Biol., 426, 2692-2701

19. Venselaar, H., te Beek. T. A. H, Kuipers, R. K. P., Hekkelman, M. L., Vriend, G. (2010) Protein structure analysis of mutations causing inheritable diseases. An e-Science approach with life scientist friendly interfaces. BMC Bioinformatics. 11, 548

20. Mitchell, A., Chang, H.-Y., Daugherty, L., Fraser, M., Hunter, S., Lopez, R., McAnulla, C., McMenamin, C., Nuka, G., Pesseat, S., et al. (2015) The InterPro protein families database: the classification resource after 15 years. Nucleic Acids Res., 43, D213-D221

21. Laskowski, R. A. and Swindells, M. B. (2011) LigPlot +: multiple ligand-protein interaction diagrams for drug discovery. J. Chem. Inf. Model., 51, 2778-2786

22. Esquela-Kerscher, A. and Slack, F. J. (2006) Oncomirs- microRNAs with a role in cancer. Nat. Rev. Cancer, 6, 259-269

23. Xu, X.-M., Wang, X.-B., Chen, M.-M., Liu, T., Li, Y.-X., Jia, W.H., Liu, M., Li, X. and Tang, H. (2012) MicroRNA-19a and -19b regulate cervical carcinoma cell proliferation and invasion by targeting CUL5. Cancer Lett., 322, 148-158

24. Wu, Q., Yang, Z., An, Y., Hu, H., Yin, J., Zhang, P., Nie, Y., Wu, K., Shi, Y. and Fan, D. (2014) MiR-19a/b modulate the metastasis of gastric cancer cells by targeting the tumour suppressor MXD1. Cell Death Dis., 5, e1144

25. Calin, G. A., Sevignani, C., Dumitru, C. D., Hyslop, T., Noch, E., Yendamuri, S., Shimizu, M., Rattan, S., Bullrich, F., Negrini, M., et al. (2004) Human microRNA genes are frequently located at fragile sites and genomic regions involved in cancers. Proc. Natl. Acad. Sci. USA, 101, 2999-3004

26. Cimmino, A., Calin, G. A., Fabbri, M., Iorio, M. V., Ferracin, M., Shimizu, M., Wojcik, S. E., Aqeilan, R. I., Zupo, S., Dono, M., et al. (2005) miR-15 and miR-16 induce apoptosis by targeting BCL2. Proc. Natl. Acad. Sci. USA, 102, 13944-13949

27. Chen, Z., Zeng, H., Guo, Y., Liu, P., Pan, H., Deng, A. and Hu, J. (2010) miRNA-145 inhibits non-small cell lung cancer cell proliferation by targeting c-Myc. J. Exp. Clin. Cancer Res., 29, 151

28. Ostenfeld, M. S., Bramsen, J. B., Lamy, P., Villadsen, S. B., Fristrup, N., Sørensen, K. D., Ulhøi, B., Borre, M., Kjems, J., Dyrskjøt, L., et al. (2010) miR-145 induces caspase-dependent and-independent cell death in urothelial cancer cell lines with targeting of an expression signature present in Ta bladder tumors. Oncogene, 29, 1073-1084

29. Feng, R., Chen, X., Yu, Y., Su, L., Yu, B., Li, J., Cai, Q., Yan, M., Liu, B. and Zhu, Z. (2010) miR-126 functions as a tumour suppressor in human gastric cancer. Cancer Lett., 298, 50-63

30. Sasahira, T., Kurihara, M., Bhawal, U. K., Ueda, N., Shimomoto, T., Yamamoto, K., Kirita, T. and Kuniyasu, H. (2012) Downregulation of miR-126 induces angiogenesis and lymphangiogenesis by activation of VEGF-A in oral cancer. Br. J. Cancer, 107, 700-706

31. Zhang, P., Ji, D.-B., Han, H.-B., Shi, Y.-F., Du, C.-Z. and Gu, J. (2014) Downregulation of miR-193a-5p correlates with lymph node metastasis and poor prognosis in colorectal cancer. World J. 
Gastroenterol., 20, 12241-12248

32. Jiang, L., Huang, Q., Zhang, S., Zhang, Q., Chang, J., Qiu, X. and Wang, E. (2010) Hsa-miR-125a-3p and hsa-miR-125a-5p are downregulated in non-small cell lung cancer and have inverse effects on invasion and migration of lung cancer cells. BMC Cancer, 10, 318

33. Johnson, S. M., Grosshans, H., Shingara, J., Byrom, M., Jarvis, R., Cheng, A., Labourier, E., Reinert, K. L., Brown, D. and Slack, F. J. (2005) RAS is regulated by the let-7 microRNA family. Cell, 120 635-647

34. Vlachos, I. S., Zagganas, K., Paraskevopoulou, M. D., Georgakilas, G., Karagkouni, D., Vergoulis, T., Dalamagas, T. and Hatzigeorgiou, A. G. (2015) DIANA-miRPath v3.0: deciphering microRNA function with experimental support. Nucleic Acids Res., 43, W460-466

35. Kolde, R. (2015). pheatmap: Pretty heatmaps [Software]. https:// CRAN.R-project.org $/$ package $=$ pheatmap

36. Green, B. D., Jabbour, A. M., Sandow, J. J., Riffkin, C. D., Masouras, D., Daunt, C. P., Salmanidis, M., Brumatti, G., Hemmings, B. A., Guthridge, M. A., et al. (2013) Akt1 is the principal Akt isoform regulating apoptosis in limiting cytokine concentrations. Cell Death Differ., 20, 1341-1349

37. Carpten, J. D., Faber, A. L., Horn, C., Donoho, G. P., Briggs, S. L., Robbins, C. M., Hostetter, G., Boguslawski, S., Moses, T. Y., Savage, S., et al. (2007) A transforming mutation in the pleckstrin homology domain of AKT1 in cancer. Nature, 448, 439-444

38. Lipkin, S. M., Wang, V., Jacoby, R., Banerjee-Basu, S., Baxevanis, A. D., Lynch, H. T., Elliott, R. M. and Collins, F. S. (2000) MLH3: a DNA mismatch repair gene associated with mammalian microsatellite instability. Nat. Genet., 24, 27-35

39. Wu, Y., Berends, M. J. W., Sijmons, R. H., Mensink, R. G. J., Verlind, E., Kooi, K. A., van der Sluis, T., Kempinga, C., van der Zee, A. G., Hollema, H., et al. (2001) A role for $M L H 3$ in hereditary nonpolyposis colorectal cancer. Nat. Genet., 29, 137138

40. Hienonen, T., Laiho, P., Salovaara, R., Mecklin, J.-P., Järvinen, H., Sistonen, P., Peltomäki, P., Lehtonen, R., Nupponen, N. N., Launonen, V., et al. (2003) Little evidence for involvement of MLH3 in colorectal cancer predisposition. Int. J. Cancer, 106, 292296

41. Korhonen, M. K., Vuorenmaa, E. and Nyström, M. (2008) The first functional study of $M L H 3$ mutations found in cancer patients. Gene. Chromosome. Canc., 47, 803-809

42. Bostrom, M. A. and Freedman, B. I. (2010) The spectrum of MYH9-associated nephropathy. Clin. J. Am. Soc. Nephrol., 5, $1107-1113$

43. Katono, K., Sato, Y., Jiang, S.-X., Kobayashi, M., Nagashio, R., Ryuge, S., Fukuda, E., Goshima, N., Satoh, Y., Saegusa, M., et al. (2015) Prognostic significance of MYH9 expression in resected non-small cell lung cancer. PLoS One, 10, e0121460

44. Mayr, C., Hemann, M. T., Bartel, D. P. (2007) Disrupting the pairing between let-7 and Hmga2 enhances oncogenic transformation. Science, 315,1576-1579

45. Young, A. R. J. and Narita, M. (2007) Oncogenic HMGA2: short or small? Genes Dev., 21, 1005-1009

46. Vaishnavi, A., Capelletti, M., Le, A. T., Kako, S., Butaney, M., Ercan, D., Mahale, S., Davies, K. D., Aisner, D. L., Pilling, A. B., et al. (2013) Oncogenic and drug-sensitive NTRK1 rearrangements in lung cancer. Nat. Med., 19, 1469-1472

47. Olive, V., Bennett, M. J., Walker, J. C., Ma, C., Jiang, I., CordonCardo, C., Li, Q.-J., Lowe, S. W., Hannon, G. J. and He, L. (2009) miR-19 is a key oncogenic component of mir-17-92. Genes Dev., 23, 2839-2849

48. Armstrong, L., Hughes, O., Yung, S., Hyslop, L., Stewart, R., Wappler, I., Peters, H., Walter, T., Stojkovic, P., Evans, J., et al. (2006) The role of PI3K/AKT, MAPK/ERK and NF $\kappa \beta$ signalling in the maintenance of human embryonic stem cell pluripotency and viability highlighted by transcriptional profiling and functional analysis. Hum. Mol. Genet., 15, 1894-1913

49. Mendoza, M. C, Er, E. E \& Blenis, J. (2011) The Ras-ERK and PI3K-mTOR pathways: cross-talk and compensation. Trends Biochem Sci., 36(6), 320-328

50. Dubrovska, A., Kim, S., Salamone, R. J., Walker, J. R., Maira, S.M., García-Echeverría, C., Schultz, P. G. and Reddy, V. A. (2009) The role of PTEN/Akt/PI3K signaling in the maintenance and viability of prostate cancer stem-like cell populations. Proc. Natl. Acad. Sci. USA, 106, 268-273

51. Carracedo, A. and Pandolfi, P. P. (2008) The PTEN-PI3K pathway: of feedbacks and cross-talks. Oncogene, 27, 5527-5541

52. Dienstmann, R., Rodon, J., Serra, V. and Tabernero, J. (2014) Picking the point of inhibition: a comparative review of PI3K/ AKT/mTOR pathway inhibitors. Mol. Cancer Ther., 13, 10211031

53. Bertacchini, J., Heidari, N., Mediani, L., Capitani, S., Shahjahani, M., Ahmadzadeh, A. and Saki, N. (2015) Targeting PI3K/AKT/ mTOR network for treatment of leukemia. Cell. Mol. Life Sci., 72, 2337-2347

54. Jameson, J. L. and Longo, D. L. (2015) Precision medicine personalized, problematic, and promising. N. Engl. J. Med., 372, 2229-2234

55. Lillie, E.O., Patay, B., Diamant, J., Issell, B., Topol, E.J., Schork, N. J. (2011) The n-of-1 clinical trial: the ultimate strategy for individualizing medicine? Pers. Med., 8, 161-173

56. Leggett, R. M., Ramirez-Gonzalez, R. H., Clavijo, B. J., Waite, D. and Davey, R. P. (2013) Sequencing quality assessment tools to enable data-driven informatics for high throughput genomics. Front. Genet., 4, 288

57. Li, H., Handsaker, B., Wysoker, A., Fennell, T., Ruan, J., Homer, N., Marth, G., Abecasis, G. and Durbin, R. (2009) The sequence alignment/map format and SAMtools. Bioinformatics, 25, 20782079

58. McKenna, A., Hanna, M., Banks, E., Sivachenko, A., Cibulskis, K., Kernytsky, A., Garimella, K., Altshuler, D., Gabriel, S., Daly, M., et al. (2010) The Genome Analysis Toolkit: a MapReduce framework for analyzing next-generation DNA sequencing data. Genome Res., 20, 1297-1303

59. Evani, U. S., Challis, D., Yu, J., Jackson, A. R., Paithankar, S., Bainbridge, M. N., Jakkamsetti, A., Pham, P., Coarfa, C., 
Milosavljevic, A., et al. (2012) Atlas2 Cloud: a framework for personal genome analysis in the cloud. BMC Genomics, 13, S19

60. Wang, K., Li, M., Hakonarson, H. (2010) ANNOVAR: functional annotation of genetic variants from high-throughput sequencing data. Nucleic Acids Res., 38, e164

61. Forbes, S. A., Beare, D., Gunasekaran, P., Leung, K., Bindal, N., Boutselakis, H., Ding, M., Bamford, S., Cole, C., Ward, S., et al. (2015) COSMIC: exploring the world's knowledge of somatic mutations in human cancer. Nucleic Acids Res., 43, D805-D811

62. Futreal, P. A., Coin, L., Marshall, M., Down, T., Hubbard, T., Wooster, R., Rahman, N. and Stratton, M. R. (2004) A census of human cancer genes. Nat. Rev. Cancer, 4, 177-183

63. Quinlan, A. R. and Hall, I. M. (2010) BEDTools: a flexible suite of utilities for comparing genomic features. Bioinformatics, 26, 841842

64. Schwarz, J. M., Rödelsperger, C., Schuelke, M. and Seelow, D. (2010) MutationTaster evaluates disease-causing potential of sequence alterations. Nat. Methods, 7, 575-576

65. Biasini M, Bienert S, Waterhouse A, Arnold K, Studer G, Schmidt T, Kiefer F, Cassarino TG, Bertoni M, Bordoli L, Schwede T. (2014). SWISS-MODEL: modelling protein tertiary and quaternary structure using evolutionary information. Nucleic Acids Res. 42,W252-W258
66. Pettersen, E. F., Goddard, T. D., Huang, C. C., Couch, G. S., Greenblatt, D. M., Meng, E. C. and Ferrin, T. E. (2004) UCSF Chimera - a visualization system for exploratory research and analysis. J. Comput. Chem., 25, 1605-1612

67. Barturen, G., Rueda, A., Hamberg, M., Alganza, A., Lebron, R., Kotsyfakis, M., Shi, B.-J., Koppers-Lalic, D. and Hackenberg, M. (2014) sRNAbench: profiling of small RNAs and its sequence variants in single or multi-species high-throughput experiments. Methods Next Gener. Seq., 1, 21-31

68. Robinson, M. D., McCarthy, D. J. and Smyth, G. K. (2010) edgeR: a Bioconductor package for differential expression analysis of digital gene expression data. Bioinformatics, 26, 139-140

69. Anders, S. and Huber, W. (2010) Differential expression analysis for sequence count data. Genome Biol., 11, R106

70. Shannon, P., Markiel, A., Ozier, O., Baliga, N. S., Wang, J. T., Ramage, D., Amin, N., Schwikowski, B. and Ideker, T. (2003) Cytoscape: a software environment for integrated models of biomolecular interaction networks. Genome Res., 13, 2498-2504

71. Hsu, S.-D., Tseng, Y.-T., Shrestha, S., Lin, Y.-L., Khaleel, A., Chou, C.-H., Chu, C.-F., Huang, H.-Y., Lin, C.-M., Ho, S.-Y., et al. (2014) miRTarBase update 2014: an information resource for experimentally validated miRNA-target interactions. Nucleic Acids Res., 42, D78-D85 